\title{
Laparoscopic Resection Combined With Aspiration for Central Renal AngiomyolipomaखPreliminary Outcomes of a Novel Surgical Technique
}

dong zhang

Ningbo City First Hospital

yue cheng

Ningbo City First Hospital

junhui jiang

Ningbo City First Hospital

zhaohu jiang

Ningbo City First Hospital

cheng zhou

Ningbo City First Hospital

Zejun Yan ( $\nabla 80291047 @ q q . c o m)$

Ningbo City First Hospital

\section{Research Article}

Keywords: Central renal angiomyolipoma, Aspiration, Laparoscopic, Surgery

Posted Date: May 5th, 2021

DOI: https://doi.org/10.21203/rs.3.rs-451423/v1

License: (1) This work is licensed under a Creative Commons Attribution 4.0 International License.

Read Full License 


\section{Abstract}

\section{Background}

The purpose of this article was to explore the safety and efficacy of laparoscopic resection combined with aspiration in a selected cohort of 17 patients with central renal angiomyolipoma(RAML).

\section{Methods}

17 patients diagnosed with central RAML by the pathological report and CT/MRI were enrolled in our center from August 2015 to November 2019. The indication for surgery was tumor size more than $4 \mathrm{~cm}$ or symptomatic tumor such as hemorrhage, backache or hematuria. The main endpoint included operative time, warm ischemia time(WIT), intraoperative blood loss, postoperative hospital time, complications and renal function.

\section{Results}

The average age was $45.3 \pm 4.8$ years, 12 patients were women, 5 patients were men. Of all cases $₫ 10$ underwent peritoneal laparoscopic resection and 7 underwent retroperitoneal surgery. All operations were performed successfully without conversion to open surgery. One patient experienced collecting system injury managed by 3-0 barbed self-retaining suture. The average size of the tumors was $(6.9 \pm 2.3) \mathrm{cm}$. The average operation time $₫$ blood loss, WIT, and postoperative hospital time were $(105.3 \pm 19.5) \mathrm{min}$, $(145.8 \pm 35.7) \mathrm{ml},(25.6 \pm 4.2) \mathrm{min}$ and $5-7$ days, respectively. No complications happened in the postoperative period, and there were no local recurrence and distant metastases at a 28.5 month followup period. Pre-operative and post-operative serum creatinine $(\mathrm{SCr})$ were $(67.6 \pm 15.8) \mu \mathrm{mol} / \mathrm{L}$ and $(65.4 \pm 16.8) \mu \mathrm{mol} / \mathrm{L}$, indicating no significant renal function drop was found. Similarly, no significant difference was found in terms of preoperative and postoperative diseased renal glomerular filtration rate \GFR囚value. The main limitation included the sample size and short follow-up time.

\section{Conclusions}

The results of this study supported that laparoscopic resection combined with aspiration was a feasible, safe and effective procedure in the treatment of RAML, especially for the large central RAML which is closely related to the collection system and renal blood vessels.

\section{Background}

RAML is a benign tumor composed of abnormally proliferating blood vessels, smooth muscles, and adipose tissue. It can be defined as central RAML if the tumor located close to the renal hilum. RAML with diameter more than $4 \mathrm{~cm}$ may rupture and hemorrhage $\mathrm{e}^{[1]}$. Therefore, surgery is recommended for these patients ${ }^{[1]}$. It is known that partial nephrectomy(PN) and selective arterial embolization(SAE) are considered superior methods for $₫ 4 \mathrm{~cm}$ or symptomatic RAML ${ }^{[2]}$. However, for central RAML, there is an intimated connection between tumor and collection system as well as renal blood vessels, which is 
associated with increased operational complications such as bleeding and urine leakag $\mathrm{e}^{[3]}$. In addition, SAE is more suitable for acute hemorrhage and is limited due to high postoperative embolism syndrome and the probability for retreatment rate ${ }^{[4]}$. Thus, it is necessary for us to make an improvement to complete satisfactory oncologic control, preserve renal function and decrease operative complications.

Preliminary reports have shown that aspiration for central RAML was feasible and safe ${ }^{[5]}$. Aspiration showed potential advantages in the safety and low recurrence rates compared with PN in the treatment of RAML, with data from Xu et al. ${ }^{[6]}$.To provide more data, further studies are still necessary. This study was performed to explore the complications, renal function change, and oncologic outcomes of laparoscopic resection combined with aspiration in the treatment of central RAML.

\section{Methods}

\section{Patients}

After ethics committee approval, data were collected from our department of urology \& nephrology and showed 17 patients with central RAML who underwent laparoscopic resection combined with aspiration between August 2015 and November 2019. All patients signed informed consent. Central RAML was defined as a tumor close to the hilar vessels or collecting system by imaging. All patients were evaluated with CTU or MRI before surgery about tumor type, size, and location. Patients were included if the imaging confirmed the central RAML was maximal fat component(CT attenuation less than $-10 \mathrm{HU}$ ) (Fig. 1A-B). The exclusion criteria were as follows: tumor was minimal fat component(CT attenuation more than $-10 \mathrm{HU})$; tumor with necrosis or calcification or cystic or hemorrhage; suspicious of renal cell cancer. Of these patients( 12 women and 5 men), 10 were treated with peritoneal laparoscopic resection and 7 were treated with retroperitoneal surgery. The main endpoint included operative time, WIT, intraoperative blood loss, postoperative hospital time, complications and renal function. The average age, $\mathrm{BMI}$ and tumor size were $\varangle 45.3 \pm 4.8 \varangle$ years, $\varangle 24.1 \pm 1.4 \rrbracket \mathrm{kg} / \mathrm{m}^{2}$ and $\varangle 6.9 \pm 2.3 \varangle \mathrm{cm}$, respectively. All patients had normal SCr and GFR based on preoperative examination.

\section{Retroperitoneal surgery}

Surgery was performed under general anesthesia in healthy lateral decubitus position. First, $10 \mathrm{~mm}, 5$ $\mathrm{mm}$, and $12 \mathrm{~mm}$ trocar were established on the cross point of the line $2 \mathrm{~cm}$ above the iliac crest, anterior line axillary and posterior axillary line below the costal margin, respectively. Second, pneumoperitoneum was established at $13 \mathrm{mmHg}$, separation of the Gerota's fascia and fat resulted in a completely exposed renal. Third, renal pedicle was freely dissected for further clamping. Based on the preoperative imaging, if the tumor was totally endogenous, intraoperative ultrasonography should be used to detect the location, size, flow signal, and the shortest distance between cortex and RAML(Fig. 2C). Then the shortest distance between cortex and RAML was marked by ultrasonic knife on the kidney surface. After making a hole in the marked renal cortex, tumor tissue was aspirated as much as possible by the suction device(0.06$0.08 \mathrm{MPa}$ ). Clamp the renal artery in case bleeding affected the surgery vision and the residual tumor was 
aspirated to dryness. On the contrary, if the tumor was exogenous, the renal artery was clamped first, then removed the section of the exogenous tumor with scissor and the remaining basal tumor was aspirated totally by the aspirator(Fig. 2A-B). In addition, if the tumor was abutting the hilar vessels, the tumor should be aspirated by suction device to prevent doctors injurying the renal vessels. Finally, renal collecting system was sewed by $3-0$ barbed self-retaining suture and renal parenchyma was sewed by 2 - 0 barbed self-retaining suture. If the wound was too wide and could not be directly sutured, the hemostatic materials were used to fill the cavity and one layer renal parenchyma suture was performed(Fig. 2D). The operation was terminated with drainage tube placed and the skin closed. Cytological examination of tumor tissue was performed in all patients.

Peritoneal surgery

Surgery was performed under general anesthesia in $70^{\circ}$ lateral decubitus position. First, a $2 \mathrm{~cm}$ incision was performed on the anterior axillary. Pneumoperitoneum was established at $13 \mathrm{mmHg}$ and a $10-\mathrm{mm}$ trocar was inserted. Then a 12-cm trocar was established on costal margin and beside the navel. It may sometimes be necessary to take another $5-\mathrm{mm}$ trocar on the posterior axillary. The renal vessels were carefully isolated. The remaining procedure was similar to the former.

Follow-Up

Amount and colour of urine and fluid from drainage bag were observed postoperatively. All patients underwent routine blood test and biochemical examination at 1 day postoperatively. CT scan was performed to observe the operative region at 4-5 days postoperatively. Then it was decided whether to pull out the drainage tube according to the CT result. CT, routine blood test, biochemical examination, GFR were checked again at 1 month postoperatively. Follow up was made by CT and biochemical examination every 6 months in the first year, followed by every year after the first year.

\section{Statistical Analysis}

STATA soft(version 12.1; StataCorp LP, College Station, TX, USA) was used to analyze the data. Student's t-test was used for continuous variable. $\mathrm{P} \bigotimes 0.05$ was considered statistically significant.

\section{Results}

Characteristics of 17 patients included age, sex, tumor location, BMI, tumor size, SCr, and GFR are listed in Table 1. The average age was $(45.3 \pm 4.8)$ years, 12 patients were women, 5 patients were men. Of all cases, 10 underwent peritoneal laparoscopic resection and 7 underwent retroperitoneal surgery. 8 patients were managed by suction and 9 patients were managed by suction combined with resection. All operations were performed successfully without conversion to open surgery. The operative and postoperative characteristics are illustrated in Table 2. There was one patient experienced collecting system injury managed by $3-0$ barbed self-retaining suture. The average operation timelblood loss, WIT, and postoperative hospital time were $(105.3 \pm 19.5) \mathrm{min},(145.8 \pm 35.7) \mathrm{ml},(25.6 \pm 4.2) \mathrm{min}$ and $5-7$ days, 
respectively. The pathologic report all showed classical RAML. No complications such as transfusion and urinary fistula happened in the postoperative period. Preoperative and postoperative 1-month $\mathrm{SCr}$ were $(67.6 \pm 15.8) \mu \mathrm{mol} / \mathrm{L}$ and $(65.4 \pm 16.8) \mu \mathrm{mol} / \mathrm{L}$, respectively, which indicated no significant renal function drop was found $(P=0.38)$. Similarly, pre-operative and post-operative diseased renal GFR were $(45.2 \pm$ $3.8) \mathrm{ml} / \mathrm{min}$ and $(43.1 \pm 4.2) \mathrm{ml} / \mathrm{min}$. This was not a significant change with $\mathrm{P}=0.068$ (Table 3 ). There were no local recurrence and distant metastases at 28.5 month follow-up period.

Table 1

Patient demographics and preoperative clinical characteristics

\begin{tabular}{|ll|}
\hline Variable & Value \\
\hline No. of patients & 17 \\
\hline Age(years), mean \pm SD & $45.3 \pm 4.8$ \\
\hline Gender & \\
\hline Male/Female & $5 / 12$ \\
\hline BMI(kg/m2), & $24.1 \pm 1.4$ \\
\hline Tumor location & \\
\hline Right/Left & $11 / 6$ \\
\hline Tumor size(cm), mean \pm SD & $6.9 \pm 2.3$ \\
\hline Preoperative Scr( $\mu$ mol/L), mean \pm SD & $67.6 \pm 15,8$ \\
\hline Preoperative diseased renal GFR(ml/min), mean \pm SD & $45.2 \pm 3.8$ \\
\hline BMl, body mass index; SCr, serum creatinine; GFR, glomerular filtration rate; SD, standard deviation. \\
\hline
\end{tabular}


Table 2

Intraoperative and postoperative findings

\begin{tabular}{|ll|}
\hline Variable & Value \\
\hline Operative time(min), mean \pm SD & $105.3 \pm 19.5$ \\
\hline Blood loss(ml), mean \pm SD & $145.8 \pm 35.7$ \\
\hline WIT(min), mean \pm SD & $25.6 \pm 4.2$ \\
\hline Injury of renal pedicle vascular & 0 \\
\hline Injury of renal collecting system & 1 \\
\hline Conversion to open surgery & 0 \\
\hline Postoperative complications & 0 \\
\hline Hospitalization days(range) & $5-7$ \\
\hline Postoperative Scr( $\mu$ mol/L), mean \pm SD & $65.4 \pm 16.8$ \\
\hline Postoperative diseased renal GFR(ml/min), mean \pm SD & $43.1 \pm 4.2$ \\
\hline Recurrence & 0 \\
\hline SD, standard deviation; SCr, serum creatinine; WIT, warm ischemia time; GFR, glomerular filtration rate. \\
\hline
\end{tabular}

Table 3

Renal function details

\begin{tabular}{|lllll|}
\hline Variable & preoperative & postoperative & t & P \\
\hline Scr( $\mu \mathrm{mol} / \mathrm{L})$, mean \pm SD & $67.6 \pm 15.8$ & $65.4 \pm 16.8$ & 0.53 & 0.38 \\
\hline Diseased renal GFR(ml/min), mean \pm SD & $45.2 \pm 3.8$ & $43.1 \pm 4.2$ & 1.53 & 0.068 \\
\hline SD, standard deviation; SCr, serum creatinine; GFR, glomerular filtration rate. & & \\
\hline
\end{tabular}

\section{Discussion}

RAML is a benign tumor composed of abnormally proliferating blood vessels, smooth muscles, and adipose tissue. With the development of imaging technology, tumor is discovered early. Active surveillance is a rational method for tumor with a diameter $₫ 4 \mathrm{~cm}^{[1]}$. While other situations such as tumor $₫ 4 \mathrm{~cm}$ or symptomatic RAML without regard to size, active and aggressive therapy is a primary curative method $^{[1]}$. Because it seems that the risk of rupture and hemorrhage is remarkable increased when tumor $₫ 4 \mathrm{~cm}$. SAE and PN are the main invasive methods. SAE is an interventional operation with the merit of less bleeding and shorter hospital stay, which is appropriate for acute bleeding and intolerant of surgical patients ${ }^{[7]}$. While points that can't be ignored are the incidence of $11-40 \%$ recurrence rate and $14-80 \%$ postoperative complications such as renal failure and hypertention ${ }^{[4]}$. In addition, European 
Association of Urology guidelines state that there is strong evidence to support the PN in the treatment of T1 renal tumor. Yet, PN also increases the risk of bleeding and urine leakage if the tumor closes to the vessels and collecting system ${ }^{[3]}$. To avoid complications, it is possible to identify anatomy carefully, which in turn, leads to prolong WIT and increases the renal function damage. On the other hand, in some cases, there is no obvious capsule boundary between the tumor and surrounding renal tissue, which increases the difficulty in removing the tumor.

During the past few years, we have explored the laparoscopic resection combined with aspiration in the treatment of central RAML. The technique was initially successfully applied to renal cysts. Similarly, adipose tissue in the RAML can be aspirated just like the liquid in the $\mathrm{cyst}^{[8]}$. Xu et al. reported that this method was safe and effective ${ }^{[5]}$. Of 10 patients, 1 patient occurred urine leakage recovered by conservative treatment finally. No other complications happened and no recurrence at a 7.7 month followup period. The mean operative time, WIT, and bleeding were $92.1 \mathrm{~min}, 27.5 \mathrm{~min}$, and $53.5 \mathrm{ml}$, respectively. Dong et al. performed an off-clamp tumor evacuation for sporadic RAML with high renal nephrometry scores, and their results were encouraging. The GFR did not change significantly at 12 month follow-up period and local recurrence and distant metastases did not occurred at 48 month follow-up period ${ }^{[9]}$. In a review of another article ${ }^{[6]}$, compared with $\mathrm{PN}$, aspiration for RAML had a similar renal function change and tumor recurrence rates. However, patients treated with aspiration had significant fewer complication rates, operative time, ischemia time, and blood loss than those underwent PN. In our study, all operations were performed successfully without conversion to open surgery. One patient experienced collecting system injury managed by $3-0$ barbed self-retaining suture. No other complications happened in the postoperative period. No significant renal function drop was found. There were no local recurrence and distant metastases at a mean follow-up of 28.5 months. Sooriakumaran et al. reported that the incidence of surgical complications was $5-23 \%$ in the treatment of renal tumor by $\mathrm{PN}^{[10-12]}$. In another study ${ }^{[13]}$, including 53 central RAML patients who underwent PN, postoperative complications occurred in 5 cases $(9.4 \%), 4$ of whom had delayed bleeding and 1 had urinary fistula. Therefore, compared with the PN, it was clear that our study completed satisfactory oncologic control, preserves renal function and decreases operative complications preferably.

In our experience, a deliberate intraoperative plan should be performed based on imaging. If the tumor is totally endogenous, resection of tumor is challenging for surgeons because of the indefinite boundary. Intraoperative ultrasonography, which can detect the location, size, flow signal, and the shortest distance between cortex and RAML is considered as a feasible tool for intraoperative tumor identification. Surgical aspiration is planned and the shortest distance between cortex and RAML is marked by ultrasonic knife on the kidney surface. After making a hole in the marked renal cortex, tumor is aspirated as much as possible by the suction device $(0.06-0.08 \mathrm{MPa})$ without clamping the renal artery. Clamp the renal artery until bleeding affected the surgery vision and the residual tumor is aspirated to dryness. Compared with PN, it can shorten WIT during the surgery, reduce the probability of renal pedicle and collecting system injury, and preserve nephrons. On the contrary, if the tumor is exogenous, the renal artery is clamped first, then removes the section of exogenous tumor with scissor and the remaining basal tumor is aspirated 
totally by the aspirator. In addition, if the tumor was abutting the hilar vessels, the tumor should be aspirated by suction device to prevent doctors injurying the renal vessels. Furthermore, in order to shorten WIT and operative time, it is necessary for us to prepare two aspirators. Because the suction device is often blocked by adipose tissue during the tumor aspiration. On the other hand, based on the preoperative imaging, ureteral catheter should be placed if the tumor closes to the collecting system. After the tumor is aspirated totally, retrograde inject diluted methylene blue into the pelvis via ureteral catheter, if methylene is found to ooze, the cavity that communicates with the collecting system should be sewed by $3-0$ barbed self-retaining suture. Additionally, it should pay attention to the sewing depth when suture the renal medulla to avoid injury of renal pedicle vessels. Three-dimension imaging is an advanced technology in the medical fields. It has the advantage of understanding the relationship between the tumor with renal pedicle and collecting system ${ }^{[14]}$. Thus, in some cases, we used this technology to understand tumor characteristics and make the surgical plan to shorter the WIT and decrease the complications.

There is a rare subtype in RAML-epithelioid angiomyolipoma(eAML), which is considered a malignant variant of RAML based on the classification of tumors by the World Health Organization in 2004. The eAML presented with mononuclear or multinuclear epithelioid cells belongs to mesenchymal tumor ${ }^{[15]}$. Previous studies revealed that eAML may occur tumor thrombus formation and distant metastasis ${ }^{[16,17]}$. Thus, laparoscopic resection combined with aspiration for eAML is not an appropriate method due to its potential malignant feature. Radiologically, eAML often presents as mass with poor or no adipose tissue $^{[18,19]}$, eAML is hyperattenuating on unenhanced $C T(\varangle 45 \mathrm{HU})$ and non-uniformed enhancement on enhanced $\mathrm{CT}^{[20]}$. In our study, patients were included if the imaging confirmed the central RAML was maximal fat component(CT attenuation less than $-10 \mathrm{HU}$ ), which is able to differentiate from eAML. A review reported that it is considered appropriate to diagnose an AML with confidence when a noncalcified, fat-containing renal mass is encountered in an adult ${ }^{[8]}$. It should be noted that 17 pathological outcomes are all typical RAML. Therefore, doctors should distinguish eAML from classic RAML to avoid misdiagnose and mistreatment according to preoperative imaging .

Some limitations should be mentioned in our study. First, our study is a single institutional retrospective design. Second, the sample size is relatively small, which might influence the clinical outcomes. Therefore, a large prospective and multicenter trial is still needed to confirm the safety and efficacy of laparoscopic resection combined with aspiration for central RAML.

\section{Conclusions}

During the follow-up 28.5 months, the central RAML patients treated with laparoscopic resection combined with aspiration appears feasible, and the complications are low. Because this method can achieve satisfactory oncologic control, preserve renal function and decrease operative complications. However, considering our limited sample and short follow-up time may affect results, long-term outcomes should be assessed by multi-center randomized trials. 


\section{Abbreviations}

RAML: Renal angiomyolipoma; WIT: Warm ischemia time; SCr: Serum creatinine; GFR: Glomerular filtration rate; PN: Partial nephrectomy; SAE: Selective arterial embolization; eAML: epithelioid angiomyolipoma

\section{Declarations}

\section{Acknowledgements}

Not applicable.

\section{Funding}

This study was financially supported by the Natural Science Foundation of Zhejiang Province(Grant No. LY18H050003 and LY17H050001) and Medical and Health Science and Technology Project of Zhejiang Province(Grant No. 2019334160).

\section{Availability of data and materials}

Not applicable.

\section{Author contributions}

$D Z$, ZJY: conceived the study and contributed in writing the manuscript; YC, JHJ, ZHJ, CZ: acquired and analyzed the data; All the authors read and approved the final version.

\section{Ethics approval and consent to participate}

Not applicable.

\section{Consent for publication}

Not applicable.

\section{Competing interests}

The author declare that they have no conflict of interest.

\section{Author details}

Department of Urology \& Nephrology, Ningbo First Hospital, The affiliated hospital of Zhejiang University. 59, Liuting Street, Ningbo, Zhejiang, China.

\section{References}


[1] Flum A S, Hamoui N, Said M A, et al. Update on the Diagnosis and Management of Renal Angiomyolipoma. J Urol. 2016,195(4):834-846. https://doi.org/ 10.1016/j.juro.2015.07.126.

[2] Kara O, Akca O, Zargar H, et al. Robotic Partial Nephrectomy in the Treatment of Renal Angiomyolipoma. J Endourol. 2016,30(3):275-279. https://doi.org/10.1089/end.2015.0624.

[3] Kim D K, Komninos C, Kim L, et al. Robot-assisted Partial Nephrectomy for Endophytic Tumors. Curr Urol Rep. 2015,16(11):1-7. https://doi.org/

$10.1007 / \mathrm{s} 11934-015-0552-4$

[4] Seyam R M, Bissada N K, Kattan S A, et al. Changing trends in presentation, diagnosis and management of renal angiomyolipoma: comparison of sporadic and tuberous sclerosis complexassociated forms. Urology. 2008,72(5):1077-1082. https://doi.org/ 10.1016/j.urology.2008.07.049.

[5] Xu B, Zhang Q, Jin J. Laparoscopic aspiration for central renal angiomyolipoma: a novel technique based on single-center initial experience. Urology. 2013,81(2):313-318.

https://doi.org/10.1016/j.urology.2012.09.047

[6] Xu B, Zhang Z N, Luo C, et al.[Comparison of safety and effectiveness between retroperitoneal laparoscopic tumor aspiration and laparoscopic partial nephrectomy in the treatment of renal angiomyolipoma]. Beijing Da Xue Xue Bao. 2018,50(4):700-704.

[7] Kiefer R M, Stavropoulos S W. The Role of Interventional Radiology Techniques in the Management of Renal Angiomyolipomas. Curr Urol Rep. 2017,18(5):36. https://doi.org/ 10.1007/s11934-017-0687-6.

[8] Jinzaki M, Silverman S G, Akita H, et al. Renal angiomyolipoma: a radiological classification and update on recent developments in diagnosis and management. Abdom Imaging. 2014,39(3):588-604. https://doi.org/ 10.1007/s00261-014-0083-3.

[9] Dong K, Shen M J, Ju G Q, et al. Off-clamp Retroperitoneoscopic Tumour Evacuation for Sporadic Renal Angiomyolipomas with High RENAL Nephrometry Scores: A Novel Surgical Technique and Its Outcomes. European Urology. 2021,79(2):283-289. https://doi.org/10.1016/j.eururo.2020.11.020.

[10] Sooriakumaran P, Gibbs P, Coughlin G, et al. Angiomyolipomata: challenges, solutions, and future prospects based on over 100 cases treated. BJU Int. 2010,105(1):101-106. https://doi.org/ 10.1111/j.1464-410X.2009.08649.x.

[11] Mues A C, Palacios J M, Haramis G, et al. Contemporary experience in the management of angiomyolipoma. J Endourol. 2010,24(11):1883-1886. https://doi.org/ 10.1089/end.2010.0223.

[12] Msezane L, Chang A, Shikanov S, et al. Laparoscopic nephron-sparing surgery in the management of angiomyolipoma: a single center experience. J Endourol. 2010,24(4):583-587. https://doi.org/ 10.1089/end.2009.0330. 
[13] Nadu A, Kleinmann N, Laufer M, et al. Laparoscopic partial nephrectomy for central tumors: analysis of perioperative outcomes and complications. J Urol. 2009,181(1):42-47. https://doi.org/ 10.1016/j.juro.2008.09.014.

[14] Li X, Yu J, Liang P, et al. Combination therapy of three-dimensional (3D) visualisation operative treatment planning system and US-guided percutaneous microwave ablation in larger renal cell carcinomas ( $D \geq 4 \mathrm{~cm}$ ): preliminary results. Int J Hyperthermia. 2017,33(3):271-277. https://doi.org/ 10.1080/02656736.2016.1247299.

[15] Tsai H Y, Lee K H, Ng K F, et al. Clinicopathologic analysis of renal epithelioid angiomyolipoma: Consecutively excised 23 cases. Kaohsiung J Med Sci. 2019,35(1):33-38. https://doi.org/ 10.1002/kjm2.12006.

[16] Lei J H, Liu L R, Wei Q, et al. A Four-Year Follow-up Study of Renal Epithelioid Angiomyolipoma: A Multi-Center Experience and Literature Review. Sci Rep. 2015,5:1-17. https://doi.org/ 10.1038/srep10030.

[17] Luo D, Gou J, Yang L, et al. Epithelioid angiomyolipoma with involvement of inferior vena cava as a tumor thrombus: a case report. Kaohsiung J Med Sci. 2011,27(2):72-75. https://doi.org/ 10.1016/j.kjms.2010.05.003.

[18] Halpenny D, Snow A, McNeill G, et al. The radiological diagnosis and treatment of renal angiomyolipoma-current status. Clin Radiol. 2010,65(2):99-108. https://doi.org/ 10.1016/j.crad.2009.09.014.

[19] Huang $K \mathrm{H}$, Huang $C Y$, Chung S D, et al. Malignant epithelioid angiomyolipoma of the kidney. J Formos Med Assoc. 2007,106(2):S51-S54. https://doi.org/ 10.1016/S0929-6646(09)60353-3.

[20] Tsukada J, Jinzaki M, Yao M, et al. Epithelioid angiomyolipoma of the kidney: radiological imaging. Int J Urol. 2013,20(11):1105-1111. https://doi.org/

10.1111/iju.12117.

\section{Figures}



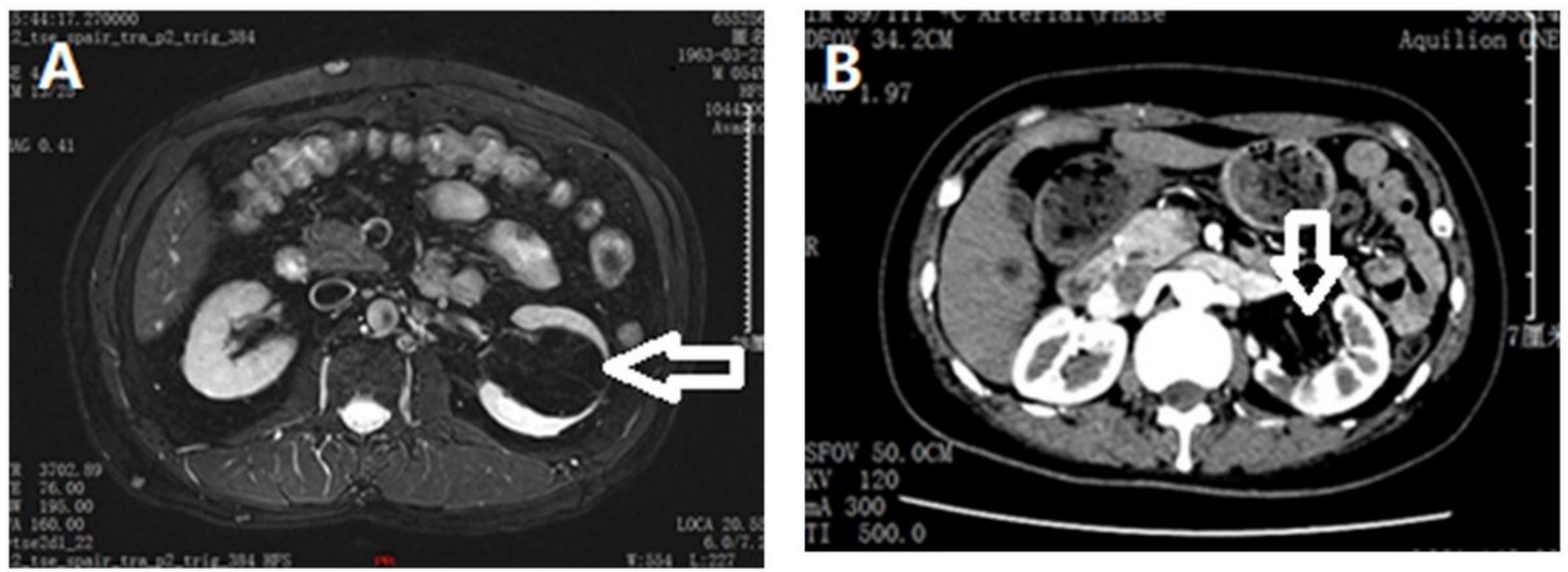

Figure 1

(A-B) Preoperative imaging confirmed the central RAML was maximal fat component(white arrow) 

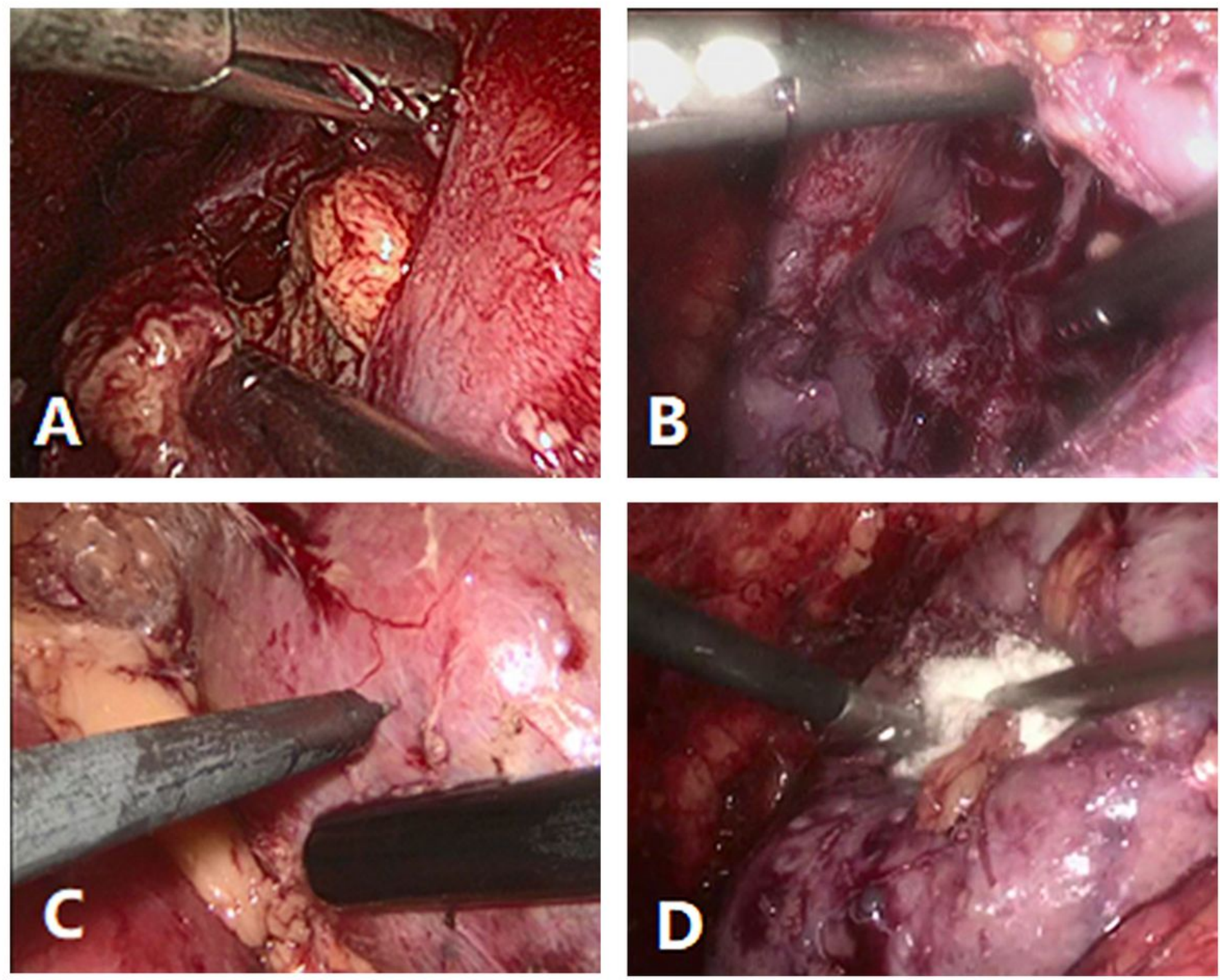

Figure 2

(A-D) Intraoperative photographs of laparoscopic resection combined with aspiration for central RAML. (A) Typical central RAML full of maximal fat component. (B) A deep cavity after the tumor aspired. (C) Intraoperative ultrasonography was used to detect the location, size, flow signal, and the shortest distance between cortex and RAML. (D) the hemostatic materials were used to fill the cavity. 